\title{
Partial Differentiation of Real Ternary Functions
}

\author{
Takao Inoué \\ Inaba 2205, Wing-Minamikan \\ Nagano, Nagano, Japan
}

\author{
Bing Xie \\ Qingdao University of Science \\ and Technology \\ China
}

\author{
Xiquan Liang \\ Qingdao University of Science \\ and Technology \\ China
}

\begin{abstract}
Summary. In this article, we shall extend the result of [19] to discuss partial differentiation of real ternary functions (refer to [8] and [16] for partial differentiation).
\end{abstract}

MML identifier: PDIFF_4, version: $7.11 .04 \quad 4.130 .1076$

The notation and terminology used here have been introduced in the following papers: [7], [12], [13], [14], [1], [2], [3], [4], [5], [8], [19], [15], [9], [18], [6], [11], [10], and [17].

\section{Preliminaries}

For simplicity, we use the following convention: $D$ denotes a set, $x, x_{0}, y, y_{0}$, $z, z_{0}, r, s, t$ denote real numbers, $p, a, u, u_{0}$ denote elements of $\mathcal{R}^{3}, f, f_{1}, f_{2}$, $f_{3}, g$ denote partial functions from $\mathcal{R}^{3}$ to $\mathbb{R}, R$ denotes a rest, and $L$ denotes a linear function.

One can prove the following three propositions:

(1) $\operatorname{dom} \operatorname{proj}(1,3)=\mathcal{R}^{3}$ and $\operatorname{rng} \operatorname{proj}(1,3)=\mathbb{R}$ and for all elements $x, y, z$ of $\mathbb{R}$ holds $(\operatorname{proj}(1,3))(\langle x, y, z\rangle)=x$. 
(2) dom $\operatorname{proj}(2,3)=\mathcal{R}^{3}$ and $\operatorname{rng} \operatorname{proj}(2,3)=\mathbb{R}$ and for all elements $x, y, z$ of $\mathbb{R}$ holds $(\operatorname{proj}(2,3))(\langle x, y, z\rangle)=y$.

(3) $\operatorname{dom} \operatorname{proj}(3,3)=\mathcal{R}^{3}$ and $\operatorname{rng} \operatorname{proj}(3,3)=\mathbb{R}$ and for all elements $x, y, z$ of $\mathbb{R}$ holds $(\operatorname{proj}(3,3))(\langle x, y, z\rangle)=z$.

\section{Partial Differentiation of Real Ternary Functions}

One can prove the following propositions:

(4) If $u=\langle x, y, z\rangle$ and $f$ is partially differentiable in $u$ w.r.t. coordinate number 1, then $\operatorname{SVF} 1(1, f, u)$ is differentiable in $x$.

(5) If $u=\langle x, y, z\rangle$ and $f$ is partially differentiable in $u$ w.r.t. coordinate number 2 , then $\operatorname{SVF} 1(2, f, u)$ is differentiable in $y$.

(6) If $u=\langle x, y, z\rangle$ and $f$ is partially differentiable in $u$ w.r.t. coordinate number 3 , then $\operatorname{SVF} 1(3, f, u)$ is differentiable in $z$.

(7) Let $f$ be a partial function from $\mathcal{R}^{3}$ to $\mathbb{R}$ and $u$ be an element of $\mathcal{R}^{3}$. Then the following statements are equivalent

(i) there exist real numbers $x_{0}, y_{0}, z_{0}$ such that $u=\left\langle x_{0}, y_{0}, z_{0}\right\rangle$ and $\operatorname{SVF} 1(1, f, u)$ is differentiable in $x_{0}$,

(ii) $\quad f$ is partially differentiable in $u$ w.r.t. coordinate number 1 .

(8) Let $f$ be a partial function from $\mathcal{R}^{3}$ to $\mathbb{R}$ and $u$ be an element of $\mathcal{R}^{3}$. Then the following statements are equivalent

(i) there exist real numbers $x_{0}, y_{0}, z_{0}$ such that $u=\left\langle x_{0}, y_{0}, z_{0}\right\rangle$ and $\operatorname{SVF} 1(2, f, u)$ is differentiable in $y_{0}$,

(ii) $\quad f$ is partially differentiable in $u$ w.r.t. coordinate number 2 .

(9) Let $f$ be a partial function from $\mathcal{R}^{3}$ to $\mathbb{R}$ and $u$ be an element of $\mathcal{R}^{3}$. Then the following statements are equivalent

(i) there exist real numbers $x_{0}, y_{0}, z_{0}$ such that $u=\left\langle x_{0}, y_{0}, z_{0}\right\rangle$ and $\operatorname{SVF} 1(3, f, u)$ is differentiable in $z_{0}$,

(ii) $\quad f$ is partially differentiable in $u$ w.r.t. coordinate number 3 .

(10) Suppose $u=\left\langle x_{0}, y_{0}, z_{0}\right\rangle$ and $f$ is partially differentiable in $u$ w.r.t. coordinate number 1 . Then there exists a neighbourhood $N$ of $x_{0}$ such that $N \subseteq \operatorname{dom} \operatorname{SVF} 1(1, f, u)$ and there exist $L, R$ such that for every $x$ such that $x \in N$ holds $(\operatorname{SVF} 1(1, f, u))(x)-(\operatorname{SVF} 1(1, f, u))\left(x_{0}\right)=$ $L\left(x-x_{0}\right)+R\left(x-x_{0}\right)$.

(11) Suppose $u=\left\langle x_{0}, y_{0}, z_{0}\right\rangle$ and $f$ is partially differentiable in $u$ w.r.t. coordinate number 2 . Then there exists a neighbourhood $N$ of $y_{0}$ such that $N \subseteq \operatorname{dom} \operatorname{SVF} 1(2, f, u)$ and there exist $L, R$ such that for every $y$ such that $y \in N$ holds $(\operatorname{SVF} 1(2, f, u))(y)-(\operatorname{SVF} 1(2, f, u))\left(y_{0}\right)=$ $L\left(y-y_{0}\right)+R\left(y-y_{0}\right)$. 
(12) Suppose $u=\left\langle x_{0}, y_{0}, z_{0}\right\rangle$ and $f$ is partially differentiable in $u$ w.r.t. coordinate number 3 . Then there exists a neighbourhood $N$ of $z_{0}$ such that $N \subseteq \operatorname{dom} \operatorname{SVF} 1(3, f, u)$ and there exist $L, R$ such that for every $z$ such that $z \in N$ holds $(\operatorname{SVF} 1(3, f, u))(z)-(\operatorname{SVF} 1(3, f, u))\left(z_{0}\right)=L\left(z-z_{0}\right)+R\left(z-z_{0}\right)$.

(13) Let $f$ be a partial function from $\mathcal{R}^{3}$ to $\mathbb{R}$ and $u$ be an element of $\mathcal{R}^{3}$. Then the following statements are equivalent

(i) $f$ is partially differentiable in $u$ w.r.t. coordinate number 1 ,

(ii) there exist real numbers $x_{0}, y_{0}, z_{0}$ such that $u=\left\langle x_{0}, y_{0}, z_{0}\right\rangle$ and there exists a neighbourhood $N$ of $x_{0}$ such that $N \subseteq \operatorname{dom} \operatorname{SVF} 1(1, f, u)$ and there exist $L, R$ such that for every $x$ such that $x \in N$ holds $(\operatorname{SVF} 1(1, f, u))(x)-$ $(\operatorname{SVF} 1(1, f, u))\left(x_{0}\right)=L\left(x-x_{0}\right)+R\left(x-x_{0}\right)$.

(14) Let $f$ be a partial function from $\mathcal{R}^{3}$ to $\mathbb{R}$ and $u$ be an element of $\mathcal{R}^{3}$. Then the following statements are equivalent

(i) $f$ is partially differentiable in $u$ w.r.t. coordinate number 2 ,

(ii) there exist real numbers $x_{0}, y_{0}, z_{0}$ such that $u=\left\langle x_{0}, y_{0}, z_{0}\right\rangle$ and there exists a neighbourhood $N$ of $y_{0}$ such that $N \subseteq \operatorname{dom} \operatorname{SVF} 1(2, f, u)$ and there exist $L, R$ such that for every $y$ such that $y \in N$ holds $(\operatorname{SVF} 1(2, f, u))(y)-$ $(\operatorname{SVF} 1(2, f, u))\left(y_{0}\right)=L\left(y-y_{0}\right)+R\left(y-y_{0}\right)$.

(15) Let $f$ be a partial function from $\mathcal{R}^{3}$ to $\mathbb{R}$ and $u$ be an element of $\mathcal{R}^{3}$. Then the following statements are equivalent

(i) $\quad f$ is partially differentiable in $u$ w.r.t. coordinate number 3 ,

(ii) there exist real numbers $x_{0}, y_{0}, z_{0}$ such that $u=\left\langle x_{0}, y_{0}, z_{0}\right\rangle$ and there exists a neighbourhood $N$ of $z_{0}$ such that $N \subseteq \operatorname{dom} \operatorname{SVF} 1(3, f, u)$ and there exist $L, R$ such that for every $z$ such that $z \in N$ holds $(\operatorname{SVF} 1(3, f, u))(z)-$ $(\operatorname{SVF} 1(3, f, u))\left(z_{0}\right)=L\left(z-z_{0}\right)+R\left(z-z_{0}\right)$.

(16) Suppose $u=\left\langle x_{0}, y_{0}, z_{0}\right\rangle$ and $f$ is partially differentiable in $u$ w.r.t. coordinate number 1 . Then $r=\operatorname{partdiff}(f, u, 1)$ if and only if there exist real numbers $x_{0}, y_{0}, z_{0}$ such that $u=\left\langle x_{0}, y_{0}, z_{0}\right\rangle$ and there exists a neighbourhood $N$ of $x_{0}$ such that $N \subseteq \operatorname{domSVF} 1(1, f, u)$ and there exist $L, R$ such that $r=L(1)$ and for every $x$ such that $x \in N$ holds $(\operatorname{SVF} 1(1, f, u))(x)-(\operatorname{SVF} 1(1, f, u))\left(x_{0}\right)=L\left(x-x_{0}\right)+R\left(x-x_{0}\right)$.

(17) Suppose $u=\left\langle x_{0}, y_{0}, z_{0}\right\rangle$ and $f$ is partially differentiable in $u$ w.r.t. coordinate number 2 . Then $r=\operatorname{partdiff}(f, u, 2)$ if and only if there exist real numbers $x_{0}, y_{0}, z_{0}$ such that $u=\left\langle x_{0}, y_{0}, z_{0}\right\rangle$ and there exists a neighbourhood $N$ of $y_{0}$ such that $N \subseteq \operatorname{domSVF} 1(2, f, u)$ and there exist $L, R$ such that $r=L(1)$ and for every $y$ such that $y \in N$ holds $(\operatorname{SVF} 1(2, f, u))(y)-(\operatorname{SVF} 1(2, f, u))\left(y_{0}\right)=L\left(y-y_{0}\right)+R\left(y-y_{0}\right)$.

(18) Suppose $u=\left\langle x_{0}, y_{0}, z_{0}\right\rangle$ and $f$ is partially differentiable in $u$ w.r.t. coordinate number 3 . Then $r=\operatorname{partdiff}(f, u, 3)$ if and only if there exist real numbers $x_{0}, y_{0}, z_{0}$ such that $u=\left\langle x_{0}, y_{0}, z_{0}\right\rangle$ and there exists a neighbourhood $N$ of $z_{0}$ such that $N \subseteq \operatorname{domSVF} 1(3, f, u)$ and there 
exist $L, R$ such that $r=L(1)$ and for every $z$ such that $z \in N$ holds $(\operatorname{SVF} 1(3, f, u))(z)-(\operatorname{SVF} 1(3, f, u))\left(z_{0}\right)=L\left(z-z_{0}\right)+R\left(z-z_{0}\right)$.

(19) If $u=\left\langle x_{0}, y_{0}, z_{0}\right\rangle$, then partdiff $(f, u, 1)=(\operatorname{SVF} 1(1, f, u))^{\prime}\left(x_{0}\right)$.

(20) If $u=\left\langle x_{0}, y_{0}, z_{0}\right\rangle$, then partdiff $(f, u, 2)=(\operatorname{SVF} 1(2, f, u))^{\prime}\left(y_{0}\right)$.

(21) If $u=\left\langle x_{0}, y_{0}, z_{0}\right\rangle$, then partdiff $(f, u, 3)=(\operatorname{SVF} 1(3, f, u))^{\prime}\left(z_{0}\right)$.

Let $f$ be a partial function from $\mathcal{R}^{3}$ to $\mathbb{R}$ and let $D$ be a set. We say that $f$ is partially differentiable w.r.t. 1st coordinate on $D$ if and only if the conditions (Def. 1) are satisfied.

(Def. 1)(i) $D \subseteq \operatorname{dom} f$, and

(ii) for every element $u$ of $\mathcal{R}^{3}$ such that $u \in D$ holds $f\lceil D$ is partially differentiable in $u$ w.r.t. coordinate number 1 .

We say that $f$ is partially differentiable w.r.t. 2nd coordinate on $D$ if and only if the conditions (Def. 2) are satisfied.

(Def. 2)(i) $\quad D \subseteq \operatorname{dom} f$, and

(ii) for every element $u$ of $\mathcal{R}^{3}$ such that $u \in D$ holds $f\lceil D$ is partially differentiable in $u$ w.r.t. coordinate number 2 .

We say that $f$ is partially differentiable w.r.t. 3rd coordinate on $D$ if and only if the conditions (Def. 3) are satisfied.

(Def. 3)(i) $D \subseteq \operatorname{dom} f$, and

(ii) for every element $u$ of $\mathcal{R}^{3}$ such that $u \in D$ holds $f\lceil D$ is partially differentiable in $u$ w.r.t. coordinate number 3 .

The following three propositions are true:

(22) Suppose $f$ is partially differentiable w.r.t. 1st coordinate on $D$. Then $D \subseteq$ $\operatorname{dom} f$ and for every $u$ such that $u \in D$ holds $f$ is partially differentiable in $u$ w.r.t. coordinate number 1 .

(23) Suppose $f$ is partially differentiable w.r.t. 2nd coordinate on $D$. Then $D \subseteq \operatorname{dom} f$ and for every $u$ such that $u \in D$ holds $f$ is partially differentiable in $u$ w.r.t. coordinate number 2 .

(24) Suppose $f$ is partially differentiable w.r.t. 3rd coordinate on $D$. Then $D \subseteq \operatorname{dom} f$ and for every $u$ such that $u \in D$ holds $f$ is partially differentiable in $u$ w.r.t. coordinate number 3 .

Let $f$ be a partial function from $\mathcal{R}^{3}$ to $\mathbb{R}$ and let $D$ be a set. Let us assume that $f$ is partially differentiable w.r.t. 1 st coordinate on $D$. The functor $f_{\uparrow D}^{1 \text { st }}$ yielding a partial function from $\mathcal{R}^{3}$ to $\mathbb{R}$ is defined as follows:

(Def. 4) $\operatorname{dom}\left(f_{\lceil D}^{1 \text { st }}\right)=D$ and for every element $u$ of $\mathcal{R}^{3}$ such that $u \in D$ holds $f_{\uparrow D}^{1 \text { st }}(u)=\operatorname{partdiff}(f, u, 1)$.

Let $f$ be a partial function from $\mathcal{R}^{3}$ to $\mathbb{R}$ and let $D$ be a set. Let us assume that $f$ is partially differentiable w.r.t. 2 nd coordinate on $D$. The functor $f_{\lceil D}^{2 \text { nd }}$ yields a partial function from $\mathcal{R}^{3}$ to $\mathbb{R}$ and is defined as follows: 
(Def. 5) $\operatorname{dom}\left(f_{\lceil D}^{2 \text { nd }}\right)=D$ and for every element $u$ of $\mathcal{R}^{3}$ such that $u \in D$ holds $f_{\lceil D}^{2 \text { nd }}(u)=\operatorname{partdiff}(f, u, 2)$.

Let $f$ be a partial function from $\mathcal{R}^{3}$ to $\mathbb{R}$ and let $D$ be a set. Let us assume that $f$ is partially differentiable w.r.t. 3 rd coordinate on $D$. The functor $f_{\uparrow D}^{3 \text { rd }}$ yielding a partial function from $\mathcal{R}^{3}$ to $\mathbb{R}$ is defined as follows:

(Def. 6) $\operatorname{dom}\left(f_{\mid D}^{3 \text { rd }}\right)=D$ and for every element $u$ of $\mathcal{R}^{3}$ such that $u \in D$ holds $f_{\lceil D}^{3 \mathrm{rd}}(u)=\operatorname{partdiff}(f, u, 3)$.

\section{Main Properties of Partial Differentiation of Real Ternary FunCTIONS}

We now state a number of propositions:

(25) Let $u_{0}$ be an element of $\mathcal{R}^{3}$ and $N$ be a neighbourhood of $(\operatorname{proj}(1,3))\left(u_{0}\right)$. Suppose $f$ is partially differentiable in $u_{0}$ w.r.t. coordinate number 1 and $N \subseteq \operatorname{domSVF} 1\left(1, f, u_{0}\right)$. Let $h$ be a convergent to 0 sequence of real numbers and $c$ be a constant sequence of real numbers. Suppose $\operatorname{rng} c=\left\{(\operatorname{proj}(1,3))\left(u_{0}\right)\right\}$ and $\operatorname{rng}(h+c) \subseteq N$. Then $h^{-1}\left(\operatorname{SVF} 1\left(1, f, u_{0}\right)\right.$. $\left.(h+c)-\operatorname{SVF} 1\left(1, f, u_{0}\right) \cdot c\right)$ is convergent and $\operatorname{partdiff}\left(f, u_{0}, 1\right)=$ $\lim \left(h^{-1}\left(\operatorname{SVF} 1\left(1, f, u_{0}\right) \cdot(h+c)-\operatorname{SVF} 1\left(1, f, u_{0}\right) \cdot c\right)\right)$.

(26) Let $u_{0}$ be an element of $\mathcal{R}^{3}$ and $N$ be a neighbourhood of $(\operatorname{proj}(2,3))\left(u_{0}\right)$. Suppose $f$ is partially differentiable in $u_{0}$ w.r.t. coordinate number 2 and $N \subseteq \operatorname{domSVF} 1\left(2, f, u_{0}\right)$. Let $h$ be a convergent to 0 sequence of real numbers and $c$ be a constant sequence of real numbers. Suppose $\operatorname{rng} c=\left\{(\operatorname{proj}(2,3))\left(u_{0}\right)\right\}$ and $\operatorname{rng}(h+c) \subseteq N$. Then $h^{-1}\left(\operatorname{SVF} 1\left(2, f, u_{0}\right)\right.$. $\left.(h+c)-\operatorname{SVF} 1\left(2, f, u_{0}\right) \cdot c\right)$ is convergent and $\operatorname{partdiff}\left(f, u_{0}, 2\right)=$ $\lim \left(h^{-1}\left(\operatorname{SVF} 1\left(2, f, u_{0}\right) \cdot(h+c)-\operatorname{SVF} 1\left(2, f, u_{0}\right) \cdot c\right)\right)$.

(27) Let $u_{0}$ be an element of $\mathcal{R}^{3}$ and $N$ be a neighbourhood of $(\operatorname{proj}(3,3))\left(u_{0}\right)$. Suppose $f$ is partially differentiable in $u_{0}$ w.r.t. coordinate number 3 and $N \subseteq \operatorname{dom} \operatorname{SVF} 1\left(3, f, u_{0}\right)$. Let $h$ be a convergent to 0 sequence of real numbers and $c$ be a constant sequence of real numbers. Suppose $\operatorname{rng} c=\left\{(\operatorname{proj}(3,3))\left(u_{0}\right)\right\}$ and $\operatorname{rng}(h+c) \subseteq N$. Then $h^{-1}\left(\operatorname{SVF} 1\left(3, f, u_{0}\right)\right.$. $\left.(h+c)-\operatorname{SVF} 1\left(3, f, u_{0}\right) \cdot c\right)$ is convergent and $\operatorname{partdiff}\left(f, u_{0}, 3\right)=$ $\lim \left(h^{-1}\left(\operatorname{SVF} 1\left(3, f, u_{0}\right) \cdot(h+c)-\operatorname{SVF} 1\left(3, f, u_{0}\right) \cdot c\right)\right)$.

(28) Suppose that

(i) $\quad f_{1}$ is partially differentiable in $u_{0}$ w.r.t. coordinate number 1 , and

(ii) $\quad f_{2}$ is partially differentiable in $u_{0}$ w.r.t. coordinate number 1 .

Then $f_{1} f_{2}$ is partially differentiable in $u_{0}$ w.r.t. coordinate number 1 .

(29) Suppose that

(i) $\quad f_{1}$ is partially differentiable in $u_{0}$ w.r.t. coordinate number 2 , and

(ii) $f_{2}$ is partially differentiable in $u_{0}$ w.r.t. coordinate number 2 . 
Then $f_{1} f_{2}$ is partially differentiable in $u_{0}$ w.r.t. coordinate number 2 .

(30) Suppose that

(i) $\quad f_{1}$ is partially differentiable in $u_{0}$ w.r.t. coordinate number 3 , and

(ii) $\quad f_{2}$ is partially differentiable in $u_{0}$ w.r.t. coordinate number 3.

Then $f_{1} f_{2}$ is partially differentiable in $u_{0}$ w.r.t. coordinate number 3 .

(31) Let $u_{0}$ be an element of $\mathcal{R}^{3}$. Suppose $f$ is partially differentiable in $u_{0}$ w.r.t. coordinate number 1 . Then $\operatorname{SVF} 1\left(1, f, u_{0}\right)$ is continuous in $(\operatorname{proj}(1,3))\left(u_{0}\right)$.

(32) Let $u_{0}$ be an element of $\mathcal{R}^{3}$. Suppose $f$ is partially differentiable in $u_{0}$ w.r.t. coordinate number 2 . Then $\operatorname{SVF} 1\left(2, f, u_{0}\right)$ is continuous in $(\operatorname{proj}(2,3))\left(u_{0}\right)$.

(33) Let $u_{0}$ be an element of $\mathcal{R}^{3}$. Suppose $f$ is partially differentiable in $u_{0}$ w.r.t. coordinate number 3 . Then $\operatorname{SVF} 1\left(3, f, u_{0}\right)$ is continuous in $(\operatorname{proj}(3,3))\left(u_{0}\right)$.

(34) Suppose $f$ is partially differentiable in $u_{0}$ w.r.t. coordinate number 1 . Then there exists $R$ such that $R(0)=0$ and $R$ is continuous in 0 .

(35) Suppose $f$ is partially differentiable in $u_{0}$ w.r.t. coordinate number 2 . Then there exists $R$ such that $R(0)=0$ and $R$ is continuous in 0 .

(36) Suppose $f$ is partially differentiable in $u_{0}$ w.r.t. coordinate number 3 . Then there exists $R$ such that $R(0)=0$ and $R$ is continuous in 0 .

\section{Grads and Curl}

Let $f$ be a partial function from $\mathcal{R}^{3}$ to $\mathbb{R}$ and let $p$ be an element of $\mathcal{R}^{3}$. The functor $\operatorname{grad}(f, p)$ yields an element of $\mathcal{R}^{3}$ and is defined as follows:

(Def. 7$) \quad \operatorname{grad}(f, p)=\operatorname{partdiff}(f, p, 1) \cdot e_{1}+\operatorname{partdiff}(f, p, 2) \cdot e_{2}+\operatorname{partdiff}(f, p, 3) \cdot e_{3}$.

We now state several propositions:

(37) $\operatorname{grad}(f, p)=[\operatorname{partdiff}(f, p, 1), \operatorname{partdiff}(f, p, 2), \operatorname{partdiff}(f, p, 3)]$.

(38) Suppose that

(i) $\quad f$ is partially differentiable in $p$ w.r.t. coordinate number 1 , partially differentiable in $p$ w.r.t. coordinate number 2 , and partially differentiable in $p$ w.r.t. coordinate number 3 , and

(ii) $\quad g$ is partially differentiable in $p$ w.r.t. coordinate number 1 , partially differentiable in $p$ w.r.t. coordinate number 2 , and partially differentiable in $p$ w.r.t. coordinate number 3 .

Then $\operatorname{grad}(f+g, p)=\operatorname{grad}(f, p)+\operatorname{grad}(g, p)$.

(39) Suppose that

(i) $\quad f$ is partially differentiable in $p$ w.r.t. coordinate number 1 , partially differentiable in $p$ w.r.t. coordinate number 2 , and partially differentiable in $p$ w.r.t. coordinate number 3 , and 
(ii) $\quad g$ is partially differentiable in $p$ w.r.t. coordinate number 1 , partially differentiable in $p$ w.r.t. coordinate number 2 , and partially differentiable in $p$ w.r.t. coordinate number 3 .

Then $\operatorname{grad}(f-g, p)=\operatorname{grad}(f, p)-\operatorname{grad}(g, p)$.

(40) Suppose that

(i) $f$ is partially differentiable in $p$ w.r.t. coordinate number 1 ,

(ii) $\quad f$ is partially differentiable in $p$ w.r.t. coordinate number 2 , and

(iii) $\quad f$ is partially differentiable in $p$ w.r.t. coordinate number 3.

Then $\operatorname{grad}(r f, p)=r \cdot \operatorname{grad}(f, p)$.

(41) Suppose that

(i) $\quad f$ is partially differentiable in $p$ w.r.t. coordinate number 1 , partially differentiable in $p$ w.r.t. coordinate number 2 , and partially differentiable in $p$ w.r.t. coordinate number 3 , and

(ii) $g$ is partially differentiable in $p$ w.r.t. coordinate number 1 , partially differentiable in $p$ w.r.t. coordinate number 2 , and partially differentiable in $p$ w.r.t. coordinate number 3 .

Then $\operatorname{grad}(s f+t g, p)=s \cdot \operatorname{grad}(f, p)+t \cdot \operatorname{grad}(g, p)$.

(42) Suppose that

(i) $f$ is partially differentiable in $p$ w.r.t. coordinate number 1 , partially differentiable in $p$ w.r.t. coordinate number 2 , and partially differentiable in $p$ w.r.t. coordinate number 3 , and

(ii) $\quad g$ is partially differentiable in $p$ w.r.t. coordinate number 1 , partially differentiable in $p$ w.r.t. coordinate number 2 , and partially differentiable in $p$ w.r.t. coordinate number 3 .

Then $\operatorname{grad}(s f-t g, p)=s \cdot \operatorname{grad}(f, p)-t \cdot \operatorname{grad}(g, p)$.

(43) If $f$ is total and constant, then $\operatorname{grad}(f, p)=0_{\mathcal{E}_{\mathrm{T}}^{3}}$.

Let $a$ be an element of $\mathcal{R}^{3}$. The functor unitvector $a$ yields an element of $\mathcal{R}^{3}$ and is defined as follows:

(Def. 8) unitvector $a=\left[\frac{a(1)}{\sqrt{a(1)^{2}+a(2)^{2}+a(3)^{2}}}, \frac{a(2)}{\sqrt{a(1)^{2}+a(2)^{2}+a(3)^{2}}}, \frac{a(3)}{\sqrt{a(1)^{2}+a(2)^{2}+a(3)^{2}}}\right]$.

Let $f$ be a partial function from $\mathcal{R}^{3}$ to $\mathbb{R}$ and let $p, a$ be elements of $\mathcal{R}^{3}$. The functor Directiondiff $(f, p, a)$ yielding a real number is defined by:

(Def. 9) Directiondiff $(f, p, a)=\operatorname{partdiff}(f, p, 1) \cdot($ unitvector $a)(1)+\operatorname{partdiff}(f, p, 2)$. (unitvector $a)(2)+\operatorname{partdiff}(f, p, 3) \cdot($ unitvector $a)(3)$.

The following propositions are true:

(44) If $a=\left\langle x_{0}, y_{0}, z_{0}\right\rangle$, then Directiondiff $(f, p, a)=\frac{\operatorname{partdiff}(f, p, 1) \cdot x_{0}}{\sqrt{x_{0}^{2}+y_{0}^{2}+z_{0}^{2}}}+$ $\frac{\text { partdiff }(f, p, 2) \cdot y_{0}}{\sqrt{x_{0}^{2}+y_{0}^{2}+z_{0}^{2}}}+\frac{\operatorname{partdiff}(f, p, 3) \cdot z_{0}}{\sqrt{x_{0}^{2}+y_{0}^{2}+z_{0}^{2}}}$.

(45) Directiondiff $(f, p, a)=\mid(\operatorname{grad}(f, p)$, unitvector $a) \mid$.

Let $f_{1}, f_{2}, f_{3}$ be partial functions from $\mathcal{R}^{3}$ to $\mathbb{R}$ and let $p$ be an element of $\mathcal{R}^{3}$. The functor $\operatorname{curl}\left(f_{1}, f_{2}, f_{3}, p\right)$ yields an element of $\mathcal{R}^{3}$ and is defined by: 
(Def. 10) $\operatorname{curl}\left(f_{1}, f_{2}, f_{3}, p\right)=\left(\operatorname{partdiff}\left(f_{3}, p, 2\right)-\operatorname{partdiff}\left(f_{2}, p, 3\right)\right) \cdot e_{1}+$ $\left(\operatorname{partdiff}\left(f_{1}, p, 3\right)-\operatorname{partdiff}\left(f_{3}, p, 1\right)\right) \cdot e_{2}+\left(\operatorname{partdiff}\left(f_{2}, p, 1\right)-\right.$ $\left.\operatorname{partdiff}\left(f_{1}, p, 2\right)\right) \cdot e_{3}$.

Next we state the proposition

(46) $\operatorname{curl}\left(f_{1}, f_{2}, f_{3}, p\right)=\left[\operatorname{partdiff}\left(f_{3}, p, 2\right)-\operatorname{partdiff}\left(f_{2}, p, 3\right), \operatorname{partdiff}\left(f_{1}, p, 3\right)-\right.$ $\left.\operatorname{partdiff}\left(f_{3}, p, 1\right), \operatorname{partdiff}\left(f_{2}, p, 1\right)-\operatorname{partdiff}\left(f_{1}, p, 2\right)\right]$.

\section{REFERENCES}

[1] Grzegorz Bancerek and Krzysztof Hryniewiecki. Segments of natural numbers and finite sequences. Formalized Mathematics, 1(1):107-114, 1990.

[2] Czesław Byliński. Finite sequences and tuples of elements of a non-empty sets. Formalized Mathematics, 1(3):529-536, 1990.

[3] Czesław Byliński. Functions and their basic properties. Formalized Mathematics, 1(1):55$65,1990$.

[4] Czesław Byliński. Functions from a set to a set. Formalized Mathematics, 1(1):153-164, 1990.

[5] Czesław Byliński. Partial functions. Formalized Mathematics, 1(2):357-367, 1990.

[6] Czesław Byliński. The sum and product of finite sequences of real numbers. Formalized Mathematics, 1(4):661-668, 1990.

[7] Agata Darmochwał. The Euclidean space. Formalized Mathematics, 2(4):599-603, 1991.

[8] Noboru Endou, Yasunari Shidama, and Keiichi Miyajima. Partial differentiation on normed linear spaces $\mathcal{R}^{n}$. Formalized Mathematics, 15(2):65-72, 2007, doi:10.2478/v10037007-0008-5.

[9] Krzysztof Hryniewiecki. Basic properties of real numbers. Formalized Mathematics, 1(1):35-40, 1990.

[10] Jarosław Kotowicz. Convergent sequences and the limit of sequences. Formalized Mathematics, 1(2):273-275, 1990.

[11] Jarosław Kotowicz. Real sequences and basic operations on them. Formalized Mathematics, 1(2):269-272, 1990.

[12] Xiquan Liang, Piqing Zhao, and Ou Bai. Vector functions and their differentiation formulas in 3-dimensional Euclidean spaces. Formalized Mathematics, 18(1):1-10, 2010, doi: 10.2478/v10037-010-0001-2.

[13] Konrad Raczkowski and Paweł Sadowski. Real function continuity. Formalized Mathematics, 1(4):787-791, 1990.

[14] Konrad Raczkowski and Paweł Sadowski. Real function differentiability. Formalized Mathematics, 1(4):797-801, 1990.

[15] Konrad Raczkowski and Paweł Sadowski. Topological properties of subsets in real numbers. Formalized Mathematics, 1(4):777-780, 1990.

[16] Walter Rudin. Principles of Mathematical Analysis. MacGraw-Hill, 1976.

[17] Andrzej Trybulec and Czesław Byliński. Some properties of real numbers. Formalized Mathematics, 1(3):445-449, 1990.

[18] Edmund Woronowicz. Relations defined on sets. Formalized Mathematics, 1(1):181-186, 1990.

[19] Bing Xie, Xiquan Liang, and Hongwei Li. Partial differentiation of real binary functions. Formalized Mathematics, 16(4):333-338, 2008, doi:10.2478/v10037-008-0041-z.

Received November 7, 2009 